\title{
Multimode dynamics in a short-pulse THz free electron laser
}

\author{
Ruslan Chulkov, ${ }^{1}$ Vitaliy Goryashko, ${ }^{2}$ Denis D. Arslanov, ${ }^{3}$ Rienk T. Jongma, ${ }^{3}$ \\ Wim J. van der Zande, ${ }^{3}$ and Vitali Zhaunerchyk ${ }^{4, *}$ \\ ${ }^{1}$ B.I. Stepanov Institute of Physics, National Academy of Science of Belarus, \\ Nesalezhnasti Avenue 68, 220072 Minsk, Belarus \\ ${ }^{2}$ Department of Physics and Astronomy, Uppsala University, 75120 Uppsala, Sweden \\ ${ }^{3}$ Radboud University Nijmegen, Institute for Molecules and Materials, Heyendaalseweg 135, \\ NL-6525 AJ Nijmegen, The Netherlands \\ ${ }^{4}$ Department of Physics, University of Gothenburg, 41296 Gothenburg, Sweden
}

(Received 19 February 2014; published 22 May 2014)

\begin{abstract}
The interaction of waveguide modes and consequences on laser operation are studied numerically in a THz free electron laser (FEL) driven by short electron bunches. The considered FEL cavity configuration is represented by a parallel-plate waveguide extending over the complete distance between cylindrical cavity mirrors with energy out-coupling through a rectangular slit in one of the mirrors. We describe the multimode FEL cavity desynchronization dynamics and predict strong enhancement in FEL power at special cavity configurations, when modes are degenerate. Furthermore, we predict that simultaneous excitation of several waveguide modes can occur but do not negatively influence the lasing process.
\end{abstract}

DOI: 10.1103/PhysRevSTAB.17.050703

PACS numbers: $41.60 . \mathrm{Cr}$

\section{INTRODUCTION}

The terahertz $(\mathrm{THz})$ frequency range finds its way quickly in many scientific applications, in particular, biomolecular sciences and medical research [1-7]. Different $\mathrm{THz}$ sources are filling up the technological gap known as the THz gap. Free electron lasers (FELs) are powerful versatile light sources, in which the coherent light is emitted by free relativistic electrons that propagate through a periodic magnetic field created by an undulator. FELs operating at $\mathrm{THz}$ frequencies allow a generation of tunable $\mathrm{THz}$ short pulses as well as tunable continuous-wave (CW) THz light.

FELs are generally driven by radio frequency linear accelerators ( $\mathrm{rf}$ linacs). Rf linacs routinely produce high intensity electron bunches with a typical duration of a few ps. As a consequence, THz FELs driven by rf linacs operate in the large slippage regime, which means that the duration of generated optical pulses is longer than the duration of electron bunches. In such FELs various short-pulse effects are known to play an essential role [8-12].

$\mathrm{THz}$ FELs require the use of cavity waveguides, which both diminish diffraction losses and enhance the transverse spatial overlap of electron bunches and optical pulses. Operation of short-pulse waveguided FELs can significantly be affected by the waveguide dispersion, which has been investigated in our recent publications [13,14].

\footnotetext{
*Corresponding author. vitali.zhaunerchyk@physics.gu.se

Published by the American Physical Society under the terms of the Creative Commons Attribution 3.0 License. Further distribution of this work must maintain attribution to the author $(s)$ and the published article's title, journal citation, and DOI.
}

Mathematically, the optical fields can be represented as a superposition of individual waveguide modes and interplay of FEL cavity modes has been addressed in some FEL publications (see, e.g., Refs. [15-23]). The coupling between these modes can lead to undesired effects, the most severe of those are spectral gaps, i.e., when at specific wavelengths lasing is impossible. Such spectral gaps were observed for CLIO [24] and FELIX [25]. Based on numerical simulations, the gaps were explained to be due to the partial waveguides employed in these FELs [21-23], which causes mode coupling when free-space modes are coupled into the partial waveguides in the undulator. Quite recently a new THz FEL FLARE (the Free electron Laser for Advanced spectroscopy and highResolution Experiments) has been constructed at the Radboud University Nijmegen (the Netherlands) [26,27], which, in contrast to the above-mentioned IR/THz FELs, employs a complete waveguide extending over the whole distance between the cavity mirrors. Surprisingly, spectral gaps are also observed here (Fig. 1), motivating a more detailed investigation of the interplay between cavity modes for fully waveguided long-wavelength FELs.

In this paper we numerically investigate the interplay between cavity modes in a short-pulse waveguided $\mathrm{THz}$ FEL and how it affects the cavity desynchronization dynamics. The FEL cavity in our study is considered to be similar to that of FLARE and consists of two parallel plates extending between two cylindrical mirrors. Simulations of the interaction between the light and the relativistic electrons is performed with the numerical code utilized in our previous studies [13,14]. We also report the code extension for modeling cavity energy redistribution over the higher-order modes. The extension takes 


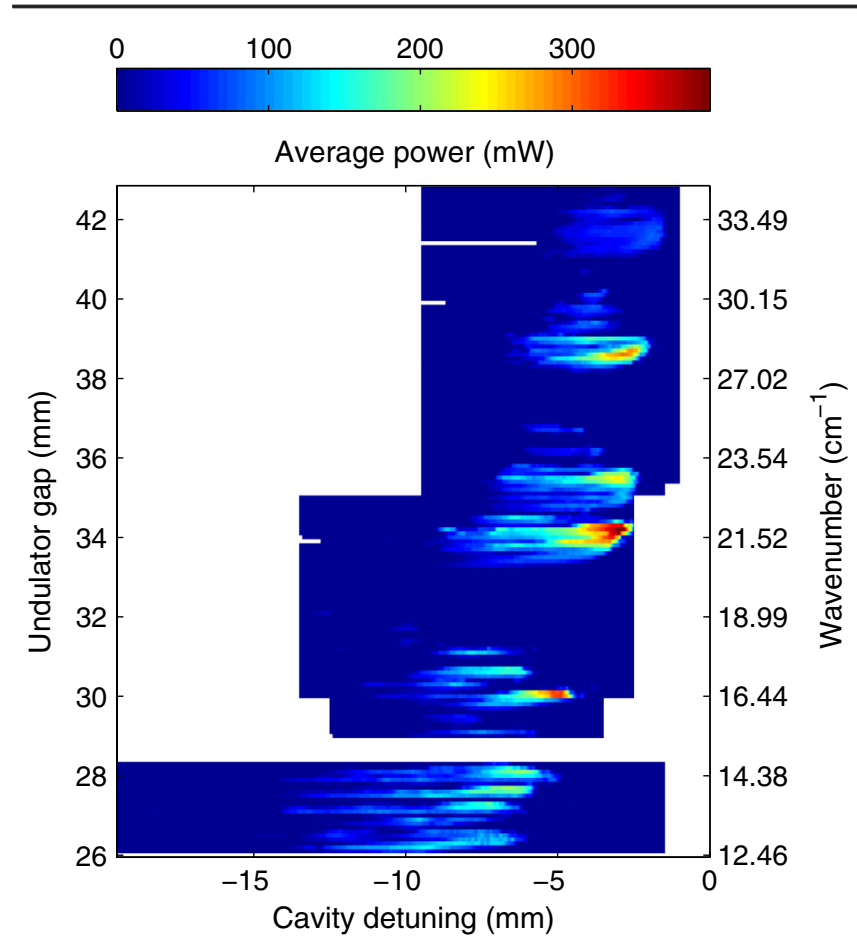

FIG. 1. 2D scan of power (false color) measured at different cavity lengths and undulator gaps. Wave numbers of the optical output as experimentally determined at the given undulator gap values are shown on the right side vertical axis. The measurements are performed at a beam energy of $15 \mathrm{MeV}$. The noncontinuous scanning of FLARE is clearly visible. The shift of the detuning curves to the longer cavity lengths with increasing wave number is due to the dispersion of the waveguide.

into account effects of the intermode scattering during light reflection from a cavity mirror possessing an outcoupling slit.

\section{FLARE DESCRIPTION}

FLARE is designed to generate $\mathrm{THz}$ light and has demonstrated lasing in the $6-100 \mathrm{~cm}^{-1}$ spectral region. The rf linac produces $10 \mu \mathrm{s}$ long trains of electron bunches with a repetition rate of $10 \mathrm{~Hz}$. Each train holds tens of thousands of 3 ps duration bunches generated at a rate of nearly $3 \mathrm{GHz}$. The FLARE cavity consists of a parallel plate waveguide that is confined by two cylindrical goldcoated aluminum mirrors. The waveguide consists of two

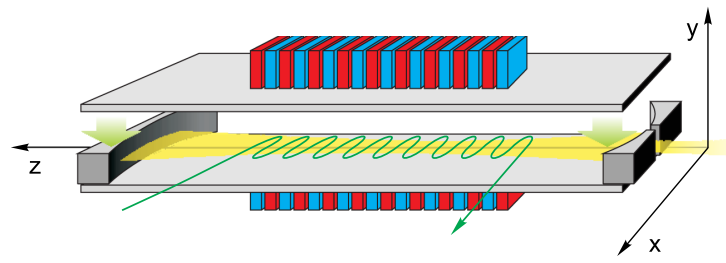

FIG. 2. Schematic of the THz FEL with the parallel plate waveguide and cylindrical mirrors.
TABLE I. FLARE parameters.

\begin{tabular}{ll}
\hline \hline rf frequency, $f_{r}$ & $2.997924 \mathrm{GHz}$ \\
Electron bunch duration & $3 \mathrm{ps}$ (standard deviation) \\
Electron bunch charge & $200 \mathrm{pC}$ \\
Undulator period & $110 \mathrm{~mm}$ \\
Number of undulator periods & 40 \\
Optical cavity length, $L_{0}$ & $7.5 \mathrm{~m}$ \\
Cavity mirrors radius, $R_{x}$ & $4.81 \mathrm{~m}$ \\
Waveguide gap, $b$ & $10 \mathrm{~mm}$ \\
Waveguide wall material & Stainless steel \\
Relative magnetic permeability & 1.008 \\
Conductivity & $1.33 \mathrm{MS} / \mathrm{m}$ \\
\hline \hline
\end{tabular}

highly polished metal plates, placed perpendicular to the undulator magnetic field, and extending over the full cavity length. The upstream cavity mirror is designed to be movable to enable cavity length detuning. The optical energy stored in the cavity is coupled out via a vertical rectangular slit in the downstream mirror. A $4.4 \mathrm{~m}$ long, the planar undulator is located in the center of the FEL cavity. The FEL geometry is schematically shown in Fig. 2 and the FLARE parameters are summarized in Table I.

\section{THE MODEL}

The FEL dynamics is simulated with a numerical code, which does not employ any averaging over the ponderomotive potential period. In particular, such a code enables dynamics modeling for FELs driven by electron bunches of comparable or even shorter length than an FEL resonant wavelength. This is the case for the part of the FLARE spectral range which is considered in this paper. In the model, the field and particle motion equations were solved numerically employing the Bashforth-Moulton four-step predictor-corrector finite-difference scheme with the precision control. The detailed model description is available elsewhere [28-31] and we describe parts that are relevant for this paper.

In the model the time-dependent electromagnetic field is expanded into a complete set of orthogonal cold parallel plate waveguide modes, $\Psi_{m, n}[16]$, which in scalar notation is described as

$$
\begin{aligned}
E(\vec{r}, t) & =2 \operatorname{Re}\left\{\sum_{m, n} \int_{0}^{\infty} C_{m, n} \Psi_{m, n} e^{i \omega t-i k_{z}^{(n)} z} d \omega\right\}, \\
H(\vec{r}, t) & =2 \operatorname{Re}\left\{\sum_{m, n} \int_{0}^{\infty} \frac{k_{z}^{(n)}}{\omega \mu_{0}} C_{m, n} \Psi_{m, n} e^{i \omega t-i k_{z}^{(n)} z} d \omega\right\},
\end{aligned}
$$

where $\vec{r}$ refers to the Cartesian coordinates with $z$ and $x, y$ being the longitudinal and the transverse coordinates, respectively, $C_{m, n}(z, \omega)$ are the slowly varying along undulator Fourier amplitudes of $\Psi_{m, n}(\vec{r})$ modes, $\omega$ is the 
angular light frequency, and $c$ is the light velocity in vacuum. The longitudinal propagation constant $k_{z}^{(n)}$ is determined as

$$
k_{z}^{(n)}(\omega)=\sqrt{\left(\frac{\omega}{c}\right)^{2}-\left(\frac{n \pi}{b}\right)^{2}},
$$

where $b$ is the distance between the waveguide plates. The $\Psi_{m, n}$ modes possess the $\pi$ or $\sigma$ polarization [16]. Since electrons wiggle perpendicular to the undulator magnetic field, the $\pi$-polarized modes, for which the direction of the electric field coincides with the wiggling motion of electrons, will predominantly be excited. The spatial profiles of the $\pi$-polarized modes are

$$
\Psi_{m, n}(\vec{r})=\frac{1}{N} X_{m, n}(x, z) Y_{n}(y), \quad \int\left|\Psi_{m, n}\right|^{2} d \vec{r}=1,
$$

where $N$ is the normalizing coefficient,

$$
\begin{aligned}
X_{m, n}(x, z)= & H_{m}\left(\sqrt{2} \frac{x}{w_{n}(z)}\right) \\
& \times \exp \left[-\frac{x^{2}}{w_{n}(z)^{2}}-i \frac{k_{z}^{(n)} x^{2}}{2 \rho_{c}(z)}+i \psi_{m}(z)\right],
\end{aligned}
$$

and

$$
Y_{n}(y)=\sqrt{\frac{2}{b}} \sin \left(\frac{n \pi}{b} y\right) .
$$

In Eqs. (5) and (6) $H_{m}$ is the Hermite polynomial of the $m$ th order, $w_{n}(z)$ is the spot size, $\rho_{c}(z)$ is the radius of curvature, $z_{R}$ is the Rayleigh length, and $\psi_{m}(z)$ is the Gouy phase shift. $z_{R}$ at FLARE is defined by the cavity mirrors radius, which is $4.81 \mathrm{~m}$, and is equal to $2 \mathrm{~m}$. The values of $w_{n}(z)$, $\rho_{c}(z)$, and $\psi_{m, n}(z)$ can be found from $z_{R}$ as

$$
\begin{aligned}
& w_{n}(z)=w_{0}^{(n)} \sqrt{1+\left(\frac{z}{z_{R}}\right)^{2}}, \quad w_{0}^{(n)}=\sqrt{\frac{2 z_{R}}{k_{z}^{(n)}}} \\
& \rho_{c}(z)=z+\frac{z_{R}^{2}}{z}, \quad \psi_{m}(z)=\frac{2 m+1}{2} \tan ^{-1}\left(\frac{z}{z_{R}}\right) .
\end{aligned}
$$

The $\Psi_{m, n}$ modes can be interpreted as a mixture of Gaussian optical modes and eigenmodes. More specifically, since there are no boundaries along the $x$ direction, the radiation field propagates as in free space and the modes in this direction have the Hermite-Gaussian amplitude profiles. In the $y$ direction, due to the waveguide boundary conditions, which imply zero tangential electric field on the waveguide surface, the radiation field is described by harmonic functions. Only the even $\Psi_{m, n}$ modes with respect to $x$, which correspond to even $m$ numbers, are excited by the electrons.

In the simulation we consider the modes with $m=0,2$, 4 , and 6 . The modes with higher $m$ are disregarded as they are significantly weaker coupled to electrons. The waveguide dispersion modifies the FEL resonant conditions and at the waist $(z=0)$ it takes the form of

$$
\frac{\omega}{v_{z}}=k_{z}^{(n)}+k_{u}+\left.\frac{d \psi_{m}(z)}{d z}\right|_{z=0},
$$

where $v_{z}$ is the average longitudinal velocity of electrons. The resonant frequencies can thus be expressed as [17]

$$
\omega_{m, n}^{r}=2 \pi c \gamma_{z} \Theta_{m}\left[1 \pm \beta_{z}\left(1-\frac{n^{2}}{4 b^{2} \Theta_{m}^{2}}\right)^{1 / 2}\right]
$$

where

$$
\Theta_{m}=\frac{\gamma_{z} \beta_{z}}{\lambda_{u}}\left(1-\frac{\lambda_{u}(m+1 / 2)}{2 \pi z_{R}}\right),
$$

$\beta_{z}=v_{z} / c$ and $\gamma_{z}$ is the Lorentz factor, $\lambda_{u}$ is the undulator period.

The boundary conditions at the cavity mirrors are taken into account through an operator of the optical feedback. This feedback is specific for each mode. The amplitude $C_{m^{\prime}, n^{\prime}}$ of the $\Psi_{m^{\prime}, n^{\prime}}$ mode at the undulator entrance and the amplitude $C_{m, n}$ of the $\Psi_{m, n}$ mode at the undulator exit at the preceding oscillator pass are related via an $\Re_{m, n}^{m^{\prime}, n^{\prime}}$ tensor as

$$
C_{m^{\prime}, n^{\prime}}=\sum_{m} \sum_{n} \Re_{m, n}^{m^{\prime}, n^{\prime}} C_{m, n}
$$

which represents the complex-value coupling coefficients. $\Re_{m, n}^{m^{\prime}, n^{\prime}}$ takes into account waveguide losses, as well as phase shifts with respect to electron bunch while the mode fields propagate through the FEL cavity and are reflected back by the mirrors:

$$
\begin{aligned}
\Re_{m, n}^{m^{\prime}, n^{\prime}}= & R_{m, n}^{m^{\prime}, n^{\prime}} \sqrt{R^{(1)} R^{(2)}}\left(1-\Gamma_{n}\right)\left(1-\Gamma_{n^{\prime}}\right) \\
& \times e^{i \omega t_{e}+i\left(\varphi_{m}+\varphi_{m^{\prime}}\right)} .
\end{aligned}
$$

In Eq. (12), $R^{(1,2)}$ are the reflection coefficients of the upstream and the downstream mirrors, $t_{e}=150 / f_{e}$ is the electron bunch period, $\varphi_{m, n}$ and $\varphi_{m^{\prime}, n^{\prime}}$ denote the phase shifts of the mode fields during the forward and backward cavity propagation, respectively, and $\Gamma_{n}$ and $\Gamma_{n^{\prime}}$ are the mode damping factors that depend on the conductivity and magnetic permeability of waveguide walls material [32]. A factor of 150 in $t_{e}$ takes into account that $150 \mathrm{THz}$ pulses simultaneously propagate in the cavity. The tensor $R_{m, n}^{m^{\prime}, n^{\prime}}$ describes scattering of the cavity modes at the downstream mirror. Its elements are determined as 


$$
R_{m, n}^{m^{\prime}, n^{\prime}}=\int \Phi(\vec{r}) \Psi_{m^{\prime}, n^{\prime}}\left(\vec{r}, \frac{L}{2}\right) \Psi_{m, n}^{*}\left(\vec{r}, \frac{L}{2}\right) d \vec{r}
$$

where $L$ is the FEL cavity length and step-wise function $\Phi(\vec{r})$ describes a reflection profile at a certain point of the downstream mirror containing the out-coupling slit, which takes zero values at points where the slit is located. The $\Phi$ function changes only in the horizontal $x$ direction, i.e., $\Phi(\vec{r}) \equiv \Phi(x)$, and is constant along the $y$ direction. Thus, all the elements $R_{m, n}^{m^{\prime}, n^{\prime}}\left(n \neq n^{\prime}\right)$ are zero which implies that the modes with different $n$ indices are noncoupled. This enables us to relate the mode amplitude coefficients at the undulator exit, $C_{m, n}^{(j)}\left(l_{u} / 2, \omega\right)$, and at the undulator entrance at the next oscillator pass, $C_{m, n}^{(j+1)}\left(-l_{u} / 2, \omega\right)$, as

$$
C_{m^{\prime}, n}^{(j+1)}\left(-l_{u} / 2, \omega\right)=\sum_{m} \Re_{m, n}^{m^{\prime}, n}(\omega) C_{m, n}^{(j)}\left(l_{u} / 2, \omega\right)
$$

where $j$ refers to a number of oscillator passes, and $l_{u}$ is the undulator length,

$$
\mathfrak{R}_{m, n}^{m^{\prime}, n}=R_{m, n}^{m^{\prime}, n} \sqrt{R^{(1)} R^{(2)}}\left(1-\tilde{\Gamma}_{n}\right) e^{i \omega t_{e}-2 i\left(k_{z}^{(n)} L-\phi_{m^{\prime}}\right)},
$$

where $\tilde{\Gamma}_{n}(\omega)$ determines the mode damping over the distance of $2 L-l_{u}$,

$$
R_{m, n}^{m^{\prime}, n}=\int \Phi(x) X_{m^{\prime}, n}\left(x, \frac{L}{2}\right) X_{m, n}^{*}\left(x, \frac{L}{2}\right) d x
$$

and

$$
\phi_{m^{\prime}}=2 \psi_{m^{\prime}}(L / 2)=\left(2 m^{\prime}+1\right) \tan ^{-1}\left(\frac{L}{2 z_{R}}\right)
$$

is the Gouy phase shift over a half cavity round trip.

The cold-cavity frequencies of the considered FEL cavity geometry (Fig. 2) are [17]

$\omega_{m, n, q}=\frac{c}{L}\left\{\left[\pi q+(2 m+1) \tan ^{-1}\left(\frac{L}{2 z_{r}}\right)\right]^{2}+\left[\frac{n \pi L}{b}\right]^{2}\right\}^{\frac{1}{2}}$,

TABLE II. Possible degenerate cavity configurations. The cavity length is $7.5 \mathrm{~m}$.

\begin{tabular}{lcc}
\hline \hline$\frac{q^{\prime}-q}{m-m^{\prime}}$ & $z_{R}, \mathrm{~m}$ & Degenerate/locked modes \\
\hline$\frac{3}{4}$ & 1.55 & $\left\{\Psi_{0,1}, \Psi_{4,1}\right\}$ and $\left\{\Psi_{2,1}, \Psi_{6,1}\right\}$ \\
$\frac{4}{6}$ & & $\left\{\Psi_{0,1}, \Psi_{6,1}\right\}$ \\
$\frac{1}{2}$ & 2.15 & $\left\{\Psi_{0,1}, \Psi_{2,1}, \Psi_{4,1}, \Psi_{6,1}\right\}$ \\
\hline \hline
\end{tabular}

TABLE III. Parameters of simulation.

\begin{tabular}{ll}
\hline \hline Mirror width & $20 \mathrm{~cm}, 40 \mathrm{~cm}$ \\
Rayleigh lengths, $z_{R}$ & $1.25-4.3 \mathrm{~m}$ \\
Mirror reflection coefficients, $R^{(1,2)}$ & 1.0 \\
Downstream mirror out-coupling rate & 0.08 \\
Magnetic flux density & $0.44 \mathrm{~T}$ \\
Electron beam energy & $11.0 \mathrm{MeV}$ \\
Resonant wavelength & $1592 \mu \mathrm{m}$ \\
\hline \hline
\end{tabular}

where $q$ is the axial index. At some cavity configurations, the cavity modes can be degenerate in frequency. Taking into account that only the modes with $n=1$ [Eq. (9)] can be resonant with the FLARE parameters considered in this paper (Table III), the modes' degeneracy for FLARE, $\omega_{m, 1, q}=\omega_{m^{\prime}, 1, q^{\prime}}$, occurs when

$$
\frac{q^{\prime}-q}{m-m^{\prime}}=\frac{2}{\pi} \tan ^{-1}\left(\frac{L}{2 z_{R}}\right)
$$

Table II lists the Rayleigh length values corresponding to three degenerate cavity configurations at the nominal cavity length of $7.5 \mathrm{~m}$. In particular, at $z_{R}=3.75 \mathrm{~m}$, where the FEL cavity is confocal, all the modes considered are degenerate.

\section{RESULTS AND DISCUSSION}

The FEL parameters used for the simulation are listed in Table III. Note that some simulation parameters do not reflect the actual design values of FLARE. The actual FLARE cavity mirror width and Rayleigh length are $20 \mathrm{~cm}$ and $2 \mathrm{~m}$, respectively, but a broader range of these parameters is considered in our study to enhance an effect of cavity mode competition and to investigate the FEL performance at degenerate cavity configurations. Figure 3 presents the saturated intracavity pulse energy calculated

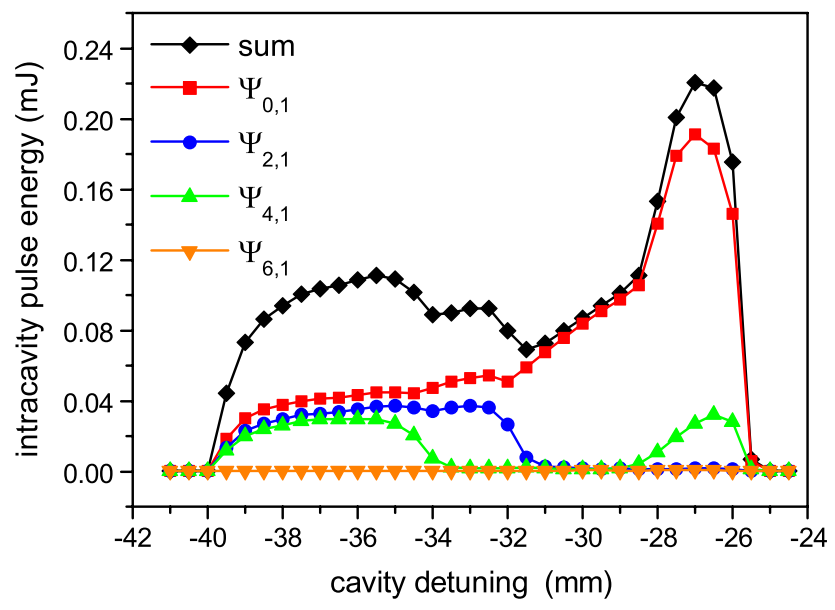

FIG. 3. Intracavity pulse energy as well as energy of most active $\Psi_{m, 1}$ modes as function of the cavity detuning at $z_{R}=2 \mathrm{~m}$. 
for the $\Psi_{m, 1}$ modes with $m=0,2,4,6$ as a function of the cavity detuning $\delta L=L-L_{0}$, where $L_{0}=7.5 \mathrm{~m}$ is the nominal cavity length. As detuning implies a shortening of the cavity, $\delta L$ is negative. The figure shows that the contribution of $\Psi_{6,1}$ as well as higher-order modes to the generated energy is negligible. The detuning curve exhibits two peaks at $\delta L=-27$ and $-35 \mathrm{~mm}$ : the first peak at $\delta L=-27 \mathrm{~mm}$ coincides with the maximum energy of the fundamental mode and the second one is broader and contains additional contributions from the $\Psi_{2,1}$ and $\Psi_{4,1}$ modes. The individual modes contribute differently to the total intracavity energy and, in particular, their contributions depend on the cavity detuning. Such a dependency can be understood in the following way: since the different $\Psi_{m, 1}$ modes resonantly interact with electrons at the frequencies following $\omega_{0,1}^{r}>\omega_{2,1}^{r}>\omega_{4,1}^{r}>\omega_{6,1}^{r}$ [Eq. (9)] and the group velocity, $v_{g}$, decreases for smaller light frequencies, shorter cavities are required to synchronize round-trip time of higher-order modes with a repetition rate of electron bunches, $f_{e}$. The mode synchronized cavity length, i.e., when $t_{e}$ coincides with the mode round-trip time, can be estimated as

$$
L_{m, 1}^{\mathrm{sync}}=\frac{v_{m, 1}^{g}}{2\left(f_{e} / 150\right)},
$$

where $v_{m, 1}^{g}$ is the group velocity at the resonant frequency of the $\Psi_{m, 1}$ mode. For the FLARE operating conditions, the modes with $m=0,2$, and 4 are expected to be synchronized with $t_{e}$ at $\delta L_{m, 1}^{\mathrm{sync}}=-24,-26$, and $-28 \mathrm{~mm}$, respectively [Eq. (20)]. However, according to the numerical results (Fig. 3), these modes appear at $\delta L_{m, 1}=-26,-32$, and $-35 \mathrm{~mm}$, respectively. The difference with respect to the $\delta L_{m, 1}^{\text {sync }}$ values is due to the slippage effect that is relevant in operation of the short-pulse FELs and leads to the maximum FEL gain shift towards larger cavity detunings by an amount of approximately few $\lambda$ (see, e.g., Ref. [14]).

At the nominal FLARE Rayleigh length of $2 \mathrm{~m}$ and the cavity length of $7.5 \mathrm{~m}$ the cavity modes are not degenerate. We have performed calculations for configurations at which modes become degenerate. This implies variation of $z_{R}$, which in practice can be achieved by varying the resonator mirror curvature. The dependencies of the saturated intracavity energy on the Rayleigh length are shown in Figs. 4 and 5. At the large detuning value, $\delta L=-38 \mathrm{~mm}$, or relatively short cavity, when all the modes considered are synchronized with the electron bunch, the total pulse energy as well as energy of the three most active modes monotonically decrease as $z_{R}$ grows (Fig. 4). The minor deviation from such a behavior is only observed at $z_{R} \approx 1.5 \mathrm{~m}$. The energy drop can be explained by an increase of the mode waist occurring at larger Rayleigh lengths [Eq. (7)] which leads to an FEL gain reduction. At the small detuning value, $\delta L=-28 \mathrm{~mm}$, the energy

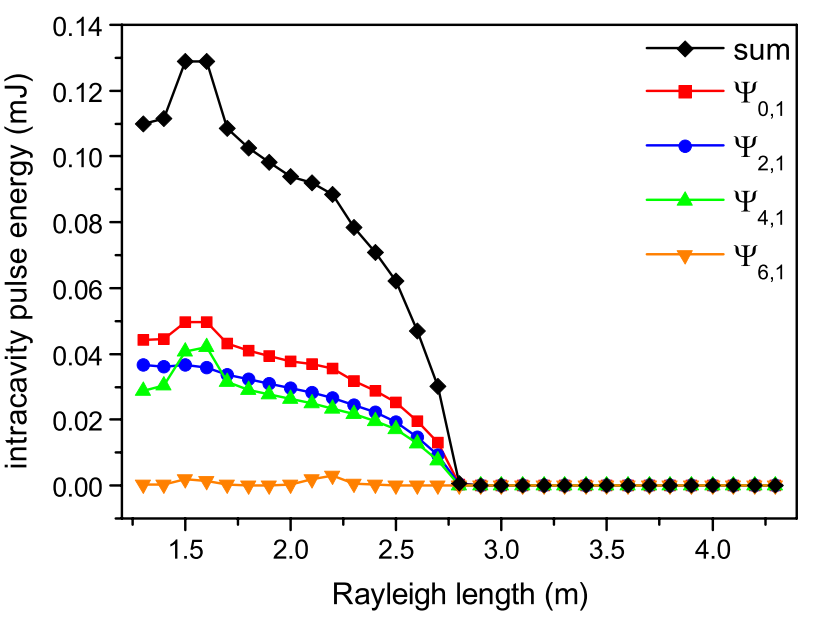

FIG. 4. Intracavity pulse energy as a function of the Rayleigh length calculated for different $\Psi_{m, 1}$ modes at $\delta L=-38 \mathrm{~mm}$.

dependence on $z_{R}$ exhibits more complex behavior (Fig. 5) and two strong peaks appear at $z_{R} \approx 1.5 \mathrm{~m}$ and $z_{R} \approx 3.8 \mathrm{~m}$ that coincide with the degenerate cavity configurations (Table II). It is surprising that at $\delta L=-28 \mathrm{~mm}$, where only the fundamental mode is synchronized with the electron bunches, the higher-order modes are excited as well. Note that excitation of higher-order modes at $L>L_{m, 1}^{\text {sync }}$ can also be observed in Fig. 3, where excitation of the $\Psi_{4,1}$ mode occurs at $\delta L=-27 \mathrm{~mm}$. In order to get insight into such an unexpected behavior we refer to the mode spectral densities discussed below.

The mode spectral densities at $\delta L=-38 \mathrm{~mm}$ and $\delta L=-28 \mathrm{~mm}$ are shown in Figs. 6 and 7, respectively, calculated for $z_{R}=1.5 \mathrm{~m}, 2.3 \mathrm{~m}$, and $3.8 \mathrm{~m}$. The case of $z_{R}=3.8 \mathrm{~m}$ at $\delta L=-38 \mathrm{~mm}$ is disregarded as no lasing occurs (Fig. 4). Far off the degenerate cavity configurations each mode predominantly oscillates at nearly its resonant frequency determined by Eq. (9) [Fig. 6(b)]. Low-intensity off-resonance peaks observed in the mode spectra are

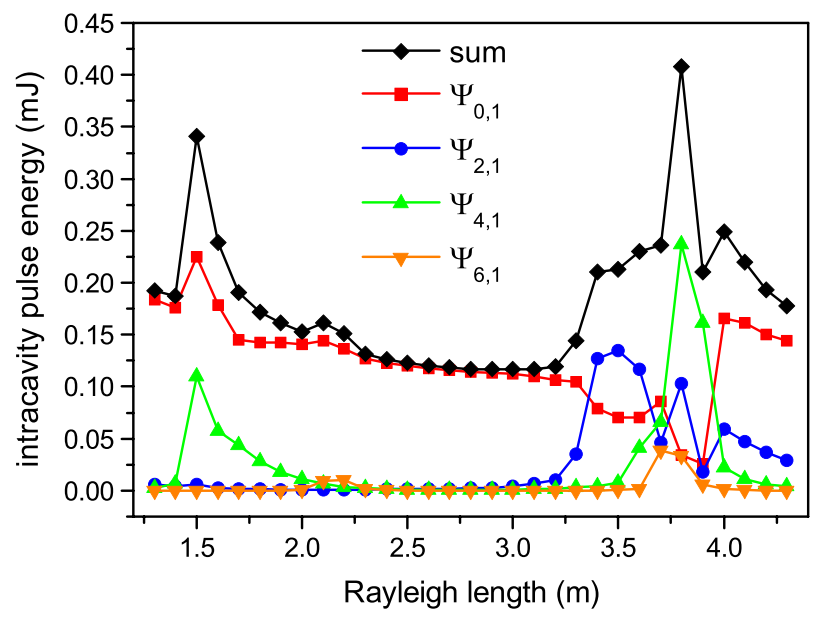

FIG. 5. The same as in Fig. 4 but when $\delta L=-28 \mathrm{~mm}$. 


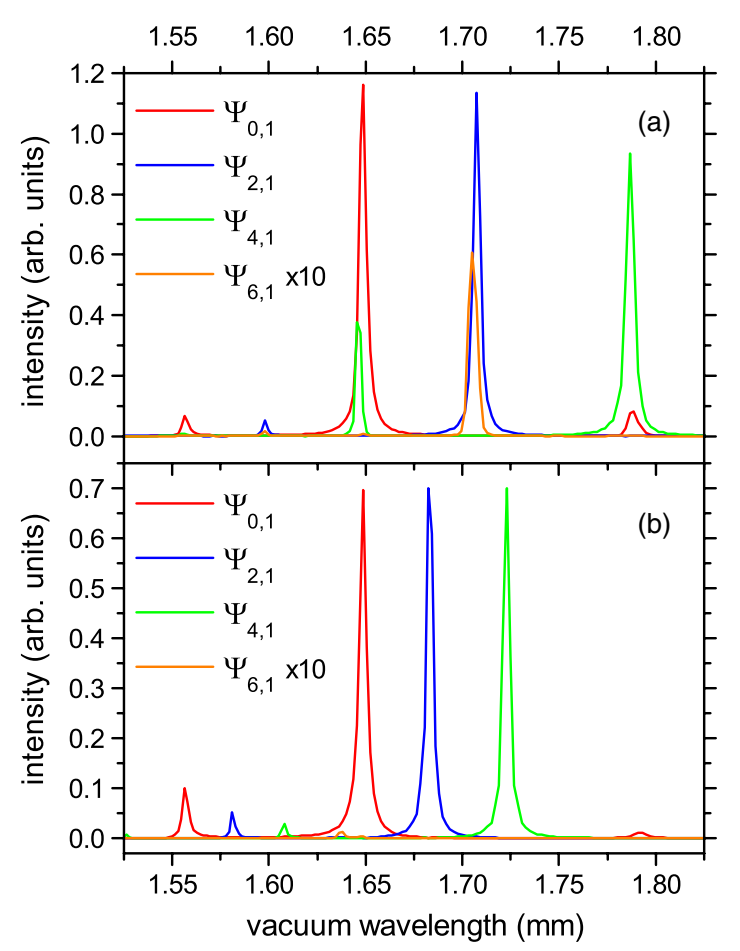

FIG. 6. The spectral density distributions of the $\Psi_{0,1}, \Psi_{2,1}, \Psi_{4,1}$, and $\Psi_{6,1}$ modes plotted at $\delta L=-38 \mathrm{~mm}$ for $z_{R}=1.5 \mathrm{~m}$ (a) and $z_{R}=2.3 \mathrm{~m}$ (b). The distributions are normalized with respect to the pulse energy.

related to modulation of the optical pulse that happens at large cavity detunings due to the reduced longitudinal overlap with electron bunches [14]. However, close to the degenerate configurations, in addition to the resonant peaks relatively strong peaks appear that coincides with the resonant frequency of the mode it is degenerate with. Since at $z_{R}=1.5 \mathrm{~m} \Psi_{0,1}$ and $\Psi_{4,1}$ are expected to be degenerate (Table II), their intensity spectra contains peaks at both $\omega_{0,1}$ and $\omega_{4,1}$ frequencies [Fig. 6(a)]. Similar behavior is also observed for the $\Psi_{6,1}$ mode, which has not been active before but appears at the resonant frequency of the $\Psi_{2,1}$ mode [Fig. 6(a)]. What we observe implies that excitation bandwidths of different modes get coupled under the condition of the mode degeneracy. This explains excitation of the higher-order modes occurring at small detuning values where only the fundamental mode can resonantly be excited by electrons [Figs. 7(a) and 7(c)].

The spectral coupling of the mode excitation bandwidths in the vicinity of the degenerate cavity configurations makes their phase locking possible. This effect is demonstrated by Figs. 8(a) and 8(b) that show the transverse spectral intensity unwrapped in time calculated at the undulator exit obtained for $z_{R}=3.5 \mathrm{~m}$ and $z_{R}=3.8 \mathrm{~m}$, respectively. When the modes are nondegenerate [Fig. 8(a)], the transverse profile of the FEL pulse is structured and exhibits fast temporally transverse intensity variations. Such variations are primarily due to the



FIG. 7. The spectral density distributions of the $\Psi_{0,1}, \Psi_{2,1}, \Psi_{4,1}$, and $\Psi_{6,1}$ modes plotted at $\delta L=-28 \mathrm{~mm}$ for $z_{R}=1.5 \mathrm{~m} \mathrm{(a),}$

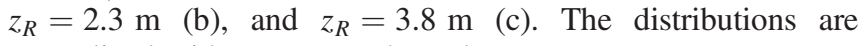
normalized with respect to the pulse energy.

interference between the $\Psi_{0,1}$ and $\Psi_{2,1}$ modes, that are the most active modes at this cavity configuration (see Fig. 5), under the condition of their relative phase being nonconstant. However, in the case of the modes' degeneracy, the transverse intensity profile is nearly steady along the entire FEL pulse and it scales with a constant factor [Fig. 8(b)]. This behavior is directly related to the phase locking of the degenerate modes. Furthermore, the modes' locking leads to a noticeable reduction of the FEL pulse duration (Fig. 8). More specifically, the FEL pulse duration at the undulator exit exceeds $230 \mathrm{ps}$ (double standard deviation) at $z_{R}=3.5 \mathrm{~m}$, while at $z_{R}=3.8 \mathrm{~m}$ it is compressed down to 80 ps. In short-pulse FELs such as FLARE, the energy saturation level is strongly dependent on the pulse duration, i.e., shorter pulse duration results in a shorter length of its interaction with electrons. Under these conditions, the single-pass FEL gain reduces but the saturated power increases [33]. Thus, the pulse 

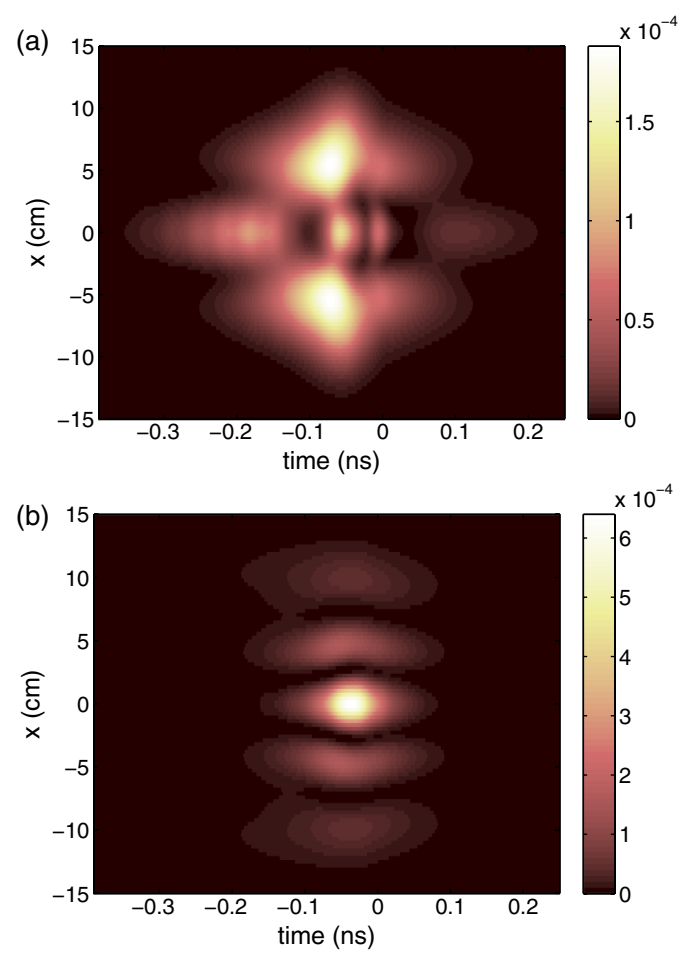

FIG. 8. The transverse temporal profiles of $\mathrm{THz}$ pulses at the undulator exit obtained at $z_{R}=3.5 \mathrm{~m}$ (a) and $z_{R}=3.8 \mathrm{~m}$ (b) with the cavity length detuned by $\delta L=-28 \mathrm{~mm}$.

compression due to the transverse mode locking can indeed lead to enhancement of the FEL energy at the degenerate cavity configurations.

The numerical data shown in Figs. 3-5 indicate that for a mirror width of $40 \mathrm{~cm}$ a single transverse mode operation can be achieved only in a narrow range of the cavity detuning values. From a practical point of view, the multimode FEL operation regime may not be desirable. This issue is of particular significance for applications in which narrow cross section and stable intensity FEL pulses are required. Physically, excitation of the high-order modes can be suppressed by inducing additional intracavity losses for these modes and the simplest is a reduction of the width of the cavity mirrors. Implementation of such mirrors increases losses for higher-order modes, e.g., the loss for the $\Psi_{6,1}$ mode increases by an order of magnitude, while the fundamental mode is unaffected. Comparing Fig. 3 with Fig. 9 confirms such a behavior. The higher-order mode contributions are suppressed with the smaller mirror and the residual energies do not exceed 10,5 , and $1 \mu \mathrm{J}$ for the $\Psi_{2,1}, \Psi_{4,1}$, and $\Psi_{6,1}$ modes, respectively.

To elaborate the conclusion that multimode interplay occurring under the conditions considered in this paper cannot lead to the spectral dips observed for FLARE, we perform addition numerical calculations for the parameters similar to Fig. 1. Figure 10 shows the line-out of Fig. 1 at $\delta L=-8 \mathrm{~mm}$ (empty gray squares) and it is compared with the simulation results. Indeed, as we can see, the numerical

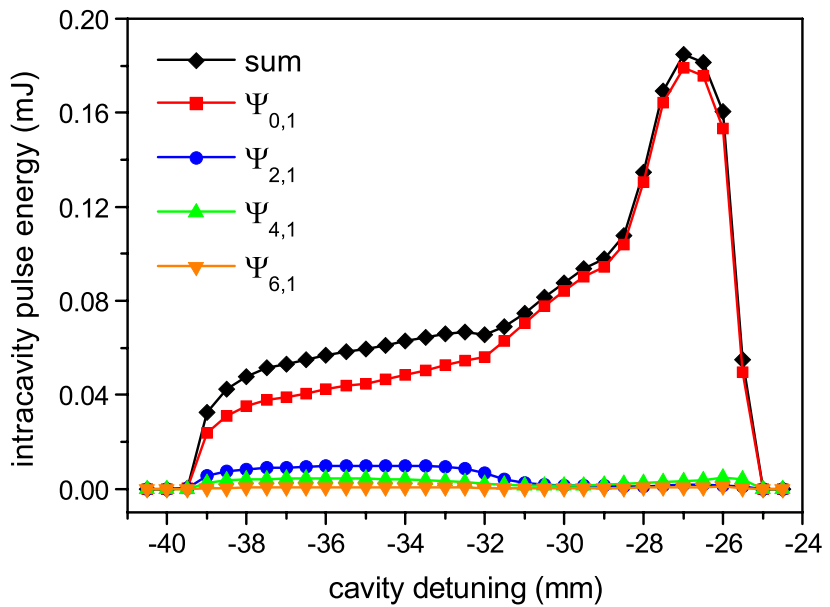

FIG. 9. Same as in Fig. 3 but when mirror width was taken to be $20 \mathrm{~cm}$.

calculations do not predict any spectral dips. As mentioned, FLARE has been designed with a full waveguide to decrease the possibility to be confronted with similar tuning problems as were observed in CLIO and in FELIX and, therefore, our simulation results supporting initial expectations are foreseen. It is important to note that, although our model does not predict the observed spectral gaps, it unambiguously points out that under the zeroemittance single-electron bunch approximation, which the simulation results are based on, the short-pulse effects and intermode scattering on the slit cannot explain the spectral gaps.

The waveguide dispersion, however, implies that the resonant conditions can be fulfilled at two resonant frequencies (see, e.g., Ref. [13]) and amplification of only

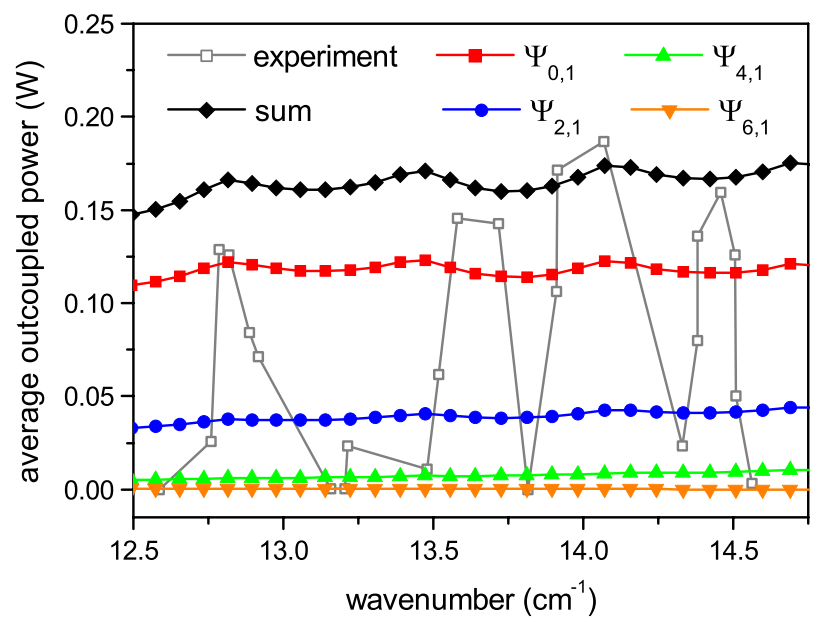

FIG. 10. FLARE average out-coupled power obtained with $15 \mathrm{MeV}$ electrons at the fixed cavity length of $7492 \mathrm{~mm}$ while scanning the undulator gap (see Fig. 1) is presented in gray. The numerical results obtained with the same parameters are shown with solid symbols. In the simulation the macropulse duration was considered to be $2 \mu \mathrm{s}$. 
the higher-frequency branch, considered in this paper, is the desired process. For FLARE, which is driven by a $3 \mathrm{GHz}$ rf linac, the cavity is actually filled with many electron bunches such that the lower-frequency branch pulses will overlap in space filling the whole cavity. Therefore, the single-bunch approximation might not be accurate enough to predict the FLARE performance and the quasi CW lowfrequency branch in the cavity possibly may compete with the higher-frequency branch. Simulation of the FEL dynamics that includes interpulse interaction is computationally substantially more demanding and may require other approximations. We will extend the code to be able to take the interaction of subsequent micropulses and the presence of both the lower- and higher-frequency resonant branches into account, hopefully enabling us to answer the question of which process leads to the observed spectral gaps.

\section{CONCLUSIONS}

In this paper we have numerically investigated various effects associated with transverse mode competition occurring in a parallel plate waveguided FEL driven by short electron bunches when the intracavity energy is outcoupled via a vertical rectangular slit mounted in a cylindrical downstream mirror. The numerical calculations have been performed for FEL parameters similar to those of FLARE. The simulations predict that at some cavity configurations the interaction between waveguide modes can lead to a significant enhancement of the $\mathrm{THz}$ power. In particular, at these cavity configurations modes become degenerate in frequency such that each degenerate mode, in addition to its own resonant frequency, is excited at the resonant frequencies of the modes it is degenerate with, i.e., the FEL operation in the vicinity of the degenerate configurations is essentially multimode and spectral bands of the excited modes are strongly coupled to each other. Such spectral coupling provides conditions for the mode locking resulting in FEL pulse compression and, respectively, in enhancement of the generated FEL energy. We also show that excitation of higher-order modes can efficiently be suppressed by reduction of the cavity mirror width, i.e., as the higher-order modes are broader in size, smaller width mirrors less efficiently reflect them.

The numerical data obtained with the actual parameters of FLARE indicate that at the long wavelength side of the FLARE spectral range no destructive mode competition occurs, neither through interaction with electron bunches nor through mode mixing on the out-coupling slit, and FLARE should essentially operate in a single transverse mode regime, which is further highlighted in Fig. 10. Based on this we can conclude that mode competition does not induce the observed spectral gaps at FLARE (Fig. 1). Alternative explanations are currently being investigated to resolve this problem.

\section{ACKNOWLEDGMENTS}

The authors would like to thank Lex van der Meer for fruitful discussions on this subject. This work was funded in part by the Swedish FEL center. V.Z. acknowledges the Swedish Research Council for providing him with the junior research fellowship grant. R. C. acknowledges the financial support from the Swedish Institute. FLARE is part of the NCAS project funded through the "Big Facilities" program of the Netherlands Organization for Scientific Research (NWO).

[1] B. Jean, Nucl. Instrum. Methods Phys. Res., Sect. A 393, 540 (1997).

[2] Y. U. Jeong, G. M. Kazakevitch, H. Jin Cha, S. Hee Park, and B. Cheol Lee, Nucl. Instrum. Methods Phys. Res., Sect. A 543, 90 (2005).

[3] D. L. Wooland, E. R. Brown, M. Pepper, and M. Kemp, Proc. IEEE 93, 1722 (2005).

[4] G. M. Png, J. W. Choi, B. W.-H. Ng, S. P. Mickan, D. Abbott, and X.-C. Zhang, Phys. Med. Biol. 53, 3501 (2008).

[5] A. R. Orlando and G. P. Gallerano, J. Infrared Milli. Terahz Waves 30, 1308 (2009).

[6] M.-A. Brun, F. Formanek, A. Yasuda, M. Sekine, N. Ando, and Y. Eishii, Phys. Med. Biol. 55, 4615 (2010).

[7] M. Kawase et al., Anal. Sci. 27, 209 (2011).

[8] D. A. Jaroszynski, D. Oepts, A. F. G. van der Meer, and P. W. van Amersfoort, Nucl. Instrum. Methods Phys. Res., Sect. A 296, 480 (1990).

[9] R. J. Bakker, C. A. J. van der Geer, D. A. Jaroszynski, A. F. G. van der Meer, D. Oepts, and P. W. van Amersfoort, Nucl. Instrum. Methods Phys. Res., Sect. A 331, 79 (1993).

[10] R. J. Bakker, C. A. J. van der Geer, D. A. Jaroszynski, A. F. G. van der Meer, D. Oepts, and P. W. van Amersfoort, J. Appl. Phys. 74, 1501 (1993).

[11] G. M. H. Knippels, R. F. X. A. M. Mols, A. F. G. van der Meer, D. Oepts, and P. W. van Amersfoort, Phys. Rev. Lett. 75, 1755 (1995).

[12] G. M. H. Knippels, A. F. G. van der Meer, R. F. X. A. M. Mols, D. Oepts, and P. W. van Amersfoort, Phys. Rev. E 53, 2778 (1996).

[13] V. Zhaunerchyk, R. T. Jongma, Yu. Lurie, Y. Pinhasi, and W. J. van der Zande, Appl. Phys. Lett. 97, 231109 (2010).

[14] V. Zhaunerchyk, D. Oepts, R. T. Jongma, and W. J. van der Zande, Phys. Rev. ST Accel. Beams 15, 050701 (2012).

[15] P. Elleame and D. A. G. Deacon, Appl. Phys. B 33, 9 (1984).

[16] L. R. Elias and J. C. Gallardo, Appl. Phys. B 31, 229 (1983).

[17] L. R. Elias, G. Ramian, J. Hu, and A. Amir, Phys. Rev. Lett. 57, 424 (1986).

[18] B. Faatz, R. W. B. Best, and P. W. van Amersfoort, Nucl. Instrum. Methods Phys. Res., Sect. A 304, 551 (1991).

[19] B. Faatz, R. W. B. Best, D. Oepts, and P. W. van Amersfoort, IEEE J. Quantum Electron. 29, 2229 (1993). 
[20] Y. Sentoku, H. Furukawa, K. Mima, T. Taguchi, S. Kuruma, H. Yasuda, C. Yamanaka, and S. Nakai, Nucl. Instrum. Methods Phys. Res., Sect. A 358, 463 (1995).

[21] R. Prazeres, F. Glotin, and J.-M. Ortega, Phys. Rev. ST Accel. Beams 12, 010701 (2009).

[22] R. Prazeres, J.-P. Berthet, F. Glotin, J.-M. Ortega, B. Biswas, and U. Kale, Phys. Rev. ST Accel. Beams 13, 090702 (2010).

[23] D. Oepts and A.F.G. van der Meer, Proceedings of FEL2010 Conference, Malmö, Sweden (Max-lab, Sweden, 2010), TUOC4.

[24] J. M. Ortega, F. Glotin, and R. Prazeres, Infrared Phys. Technol. 49, 133 (2006).

[25] L. Yi Lin and A. F. G. van der Meer, Rev. Sci. Instrum. 68, 4342 (1997).

[26] R. T. Jongma et al., Proceedings of FEL2008, Gyeongju, Korea, TUAA05.
[27] R. T. Jongma et al., Proceedings FEL2009, Liverpool, UK (STFC Daresbury Laboratory, Warrington, 2009), TUPC84.

[28] Y. Pinhasi, V. Shterngartz, and A. Gover, Phys. Rev. E 54, 6774 (1996).

[29] Y. Pinhasi, Yu. Lurie, and A. Yahalom, Nucl. Instrum. Methods Phys. Res., Sect. A 475, 147 (2001).

[30] M. J. de Loos, C. A. J. van der Geer, S. B. van der Geer, A. F. G. van der Meer, D. Oepts, and R. Wünsch, Nucl. Instrum. Methods Phys. Res., Sect. A 507, 97 (2003).

[31] V. A. Goryashko, K. Ilyenko, and A. N. Opanasenko, Nucl. Instrum. Methods Phys. Res., Sect. A 620, 462 (2010).

[32] J. D. Jackson, Classical Electrodynamics (Wiley, New York, 1999), 3rd ed., chap. 8.

[33] N. Piovella, P. Chaix, G. Shvets, and D. A. Jaroszynski, Phys. Rev. E 52, 5470 (1995). 